\title{
Three-Year Results of the SONATA Pivotal Trial of Transcervical Fibroid Ablation for Symptomatic Uterine Myomata
}

\author{
Andrea Lukes, MD, ${ }^{1}$ and Minda A. Green, MD²
}

\begin{abstract}
Objective: This article reports on 3-year clinical outcomes of the Sonography Guided Transcervical Ablation of Uterine Fibroids (SONATA) pivotal trial of transcervical fibroid ablation (TFA) in women with symptomatic uterine myomata. Materials and Methods: The SONATA, prospective, controlled, multicenter interventional trial enrolled 147 premenopausal women with symptomatic uterine fibroids who underwent uterus-preserving, sonography-guided TFA with the Sonata ${ }^{\circledR}$ System (Gynesonics, Inc., Redwood City, CA, USA). Clinical outcomes were assessed over 3 years and included surgical reinterventions, Symptom Severity Score (SSS), and Health-Related Quality of Life (HRQoL) subscales of the Uterine Fibroid Symptom and Quality-of-Life Questionnaire, EuroQol 5-Dimension (EQ-5D) questionnaire, Overall Treatment Effect, treatment satisfaction, physical activity, work impairment, pregnancy outcomes, and adverse events.

Results: The 3-year rates of surgical reintervention for heavy menstrual bleeding calculated by the binomial and Kaplan-Meier methods were $9.2 \%$ and $8.2 \%$, respectively. Compared to baseline, mean SSS decreased from $55 \pm 19$ to $22 \pm 21$, HRQoL increased from $40 \pm 21$ to $83 \pm 23$, and EQ-5D increased from $0.72 \pm 0.21$ to $0.88 \pm 0.16$ (all $p<0.001$ ). Treatment benefit on the SSS, HRQoL, and EQ-5Q exceeded the minimal clinically important difference at every follow-up visit over 3 years. At 3 years, $94 \%$ of the subjects reported treatment satisfaction, $88 \%$ reported reduced fibroid symptoms, work absenteeism due to fibroid symptoms decreased from $2.9 \%$ to $1.4 \%$, and impairment due to fibroids decreased from $51 \%$ to $12 \%$ for work, and $58 \%$ to $14 \%$ for physical activity (all $p<0.001$ ). No late complications occurred.

Conclusions: Women treated with sonography-guided TFA in the SONATA pivotal trial experienced significant and durable reduction of fibroid-related symptoms, with low surgical reintervention rates over 3 years of follow-up. ( J GYNECOL SURG 36:228)
\end{abstract}

Keywords: leiomyoma, radiofrequency ablation, SONATA, TFA, transcervical fibroid ablation, uterine fibroid

\section{Introduction}

$\mathbf{U}$ TERINE FIBROIDS ARE the most common benign pelvic tumors in women, and are present in nearly $70 \%$ of white women and more than $80 \%$ of black women in the United States prior to menopause. ${ }^{1}$ Among women with fibroids, more than one-half $(54 \%)$ report myoma-associated symptoms, such as heavy menstrual bleeding (HMB) and about one-third of women with fibroids report symptoms severe enough to have a negative impact on activities of daily living
(ADLs) such as sexual activity, work attendance and performance, and personal relationships. ${ }^{2,3}$ The combined effect of the direct costs attributable to fibroid diagnosis and treatment, plus the indirect costs due to work absenteeism and loss of productivity, are responsible for a significant economic burden of $\$ 34$ billion annually in the United States. ${ }^{4}$

A variety of treatment options are available to women with symptomatic uterine fibroids, each option with its own advantages and disadvantages. Because the etiology and location of fibroids as well as the presentation of symptoms

\footnotetext{
${ }^{1}$ Carolina Women's Research and Wellness Center, Durham, North Carolina, USA.

${ }^{2}$ Virtua OB/Gyn, Voorhees, New Jersey, USA.

(c) Andrea Lukes and Minda A Green 2020; Published by Mary Ann Liebert, Inc. This Open Access article is distributed under the terms of the Creative Commons Attribution Noncommercial License (http://creativecommons.org/licenses/by-nc/4.0/) which permits any noncommercial use, distribution, and reproduction in any medium, provided the original author(s) and the source are cited.
} 
is quite heterogeneous among affected women, the ideal therapy should be determined by shared decision-making between a woman and her gynecologist after full consideration of important factors, such as fibroid location/size, desire to preserve fertility and/or uterus, and willingness to undergo invasive surgery. In a survey of 968 women reporting symptoms caused by uterine fibroids, $79 \%$ expressed a desire for treatments that avoided invasive surgery and $51 \%$ favored treatments that preserved their uteri. ${ }^{5}$

To fill the existing uterine-fibroid treatment gap between the poor effectiveness of medical management and the invasiveness and radical approach of hysterectomy, lessinvasive methods of fibroid treatment have been developed that conserve the uterus. Radiofrequency (RF) ablationwhich can be delivered by laparoscopic, transvaginal, or transcervical approaches - has emerged as a safe and effective treatment option to fill this unmet need. ${ }^{6-9}$ A meta-analysis of 32 studies with more than 1200 patients concluded that RF ablation provided statistically significant and clinically important reductions of fibroid-related symptoms and improvements in quality of life, with low surgical reintervention rates. ${ }^{6}$ Transcervical fibroid ablation (TFA) has certain advantages that might appeal to patients, including a noninvasive delivery method, uterine preservation, ability to treat a wide range of fibroid types, avoidance of general anesthesia, and rapid recovery and return to ADLs following the procedure.

The Sonography Guided Transcervical Ablation of Uterine Fibroids (SONATA) trial was a U.S. Food and Drug Administration (FDA) Investigational Device Exemption (IDE) pivotal trial designed to assess the safety and efficacy of TFA in women with symptomatic uterine fibroids. Previously, the 1-year and 2-year results from this trial were reported. ${ }^{10,11}$ Here, this article presents the 3 -year results from the SONATA pivotal trial.

\section{Materials and Methods}

The SONATA trial was a prospective, controlled, multicenter interventional trial designed to assess the safety and efficacy of TFA for treatment of symptomatic uterine fibroids, compared to predefined objective performance criteria established by the FDA. Each participating site received institutional review board or ethics committee approval and all patients provided informed consent to participate in the trial. The clinical trial was registered at ClinicalTrials.gov (NCT02228174) and was approved by the FDA in the United States and by the Federal Commission for Protection against Health Risks (COFEPRIS) in Mexico.

The trial was specifically designed to enroll women experiencing symptoms caused by a wide range of uterine fibroid types, with each woman serving as her own control. The key inclusion criteria for the trial were premenopausal women ages 25-50 with regular and predictable menstrual cycles in order to minimize the effect of bias from rare naturally improving symptoms and to minimize inclusion of women with anovulatory abnormal uterine bleeding (AUB-O). In addition, patients had HMB with between 1 and 10 International Federation of Gynecology and $\mathrm{Ob}-$ stetrics (FIGO) type 1, 2, 3, 4, and/or 2-5 (transmural) uterine fibroids between 1 and $5 \mathrm{~cm}$ in diameter, at least 1 fibroid of which must have indented or abutted the endometrial cavity (type 1 , type 2 , type 3 or type $2-5$ ).
The key exclusion criteria were:

(1) Desire for future pregnancy

(2) Any type 0 fibroid $\geq 1.0 \mathrm{~cm}$, endometrial polyps $\geq 1.5 \mathrm{~cm}$, or multiple polyps of any size

(3) Bulk symptoms in association with subserous fibroids

(4) Previous endometrial ablation, uterine artery embolization (UAE), uterine artery occlusion, or hyperthermic ablation of fibroids

(5) Uterine volume $\geq 1000 \mathrm{~cm}^{3}$

(6) Presence of tubal implants for sterilization, or

(7) Clinically significant adenomyosis

The TFA device and procedure used in the trial was described in detail previously. ${ }^{9,12}$ TFA was performed using the Sonata ${ }^{\circledR}$ System (Gynesonics, Inc., Redwood City, CA, USA). Following the procedure, patients returned at 10 days, 30 days, 3 months, 6 months, and annually thereafter through the final follow-up visit at 3 years. The 24- and 36-month follow-up timepoints were included to gather longer-term data during the postmarket phase and were not included to support the application for FDA clearance.

The coprimary endpoint of surgical reintervention rate due to HMB was reported using Kaplan-Meier methods, with a sensitivity analysis using binomial methods wherein the event count was divided by the evaluable sample size. Secondary endpoint measurements included the symptom severity score (SSS) and health-related quality of life (HRQoL) subscales of the Uterine Fibroid Symptom and Quality-of-Life (UFS-QoL) Questionnaire. ${ }^{13}$ Scores were reported on a $0-100$ scale on which higher SSS scores indicated more-severe symptoms and lower HRQoL scores indicated worse quality of life. Change in general health status was assessed with the EuroQol 5-Dimension (EQ-5D) questionnaire. Patients self-reported their treatment benefit at 3 years as improved, no change, or worsened on the McMaster Overall Treatment Effect (OTE) scale. Treatment satisfaction was measured on a 6-item scale ranging from very satisfied to very dissatisfied. The Work Productivity and Activity Impairment Questionnaire: Specific Health Problem (WPAI:SHP) questionnaire was used to assess change in work and activity patterns following treatment. ${ }^{14}$ Adverse events (AEs) were reported according to seriousness and relationship to the device or procedure.

Safety analyses included all treated patients and efficacy analyses excluded patients who reached menopause, underwent surgical reintervention, or who withdrew from the trial during follow-up. The Wilcoxon signed-rank test was used to assess change over time for symptom severity, HRQoL, general health, and work/activity limitation outcomes.

To facilitate clinical interpretation of results, the treatment effects were also reported for SSS, HRQoL, and EQ$5 \mathrm{D}$ in standardized minimal clinically important difference (MCID) units, wherein the standardized MCID for each outcome was calculated as the mean change from baseline divided by the MCID. ${ }^{15,16}$ The published MCID for each outcome is a 10-point decrease for SSS, ${ }^{17}$ a 20-point increase for HRQoL, ${ }^{13}$ and a 0.074 -point increase for EQ-5D. ${ }^{18}$ Treatment effects below 0.5 MCID units indicate that it is unlikely that an appreciable number of patients will show a clinically important benefit. Treatment effects between 0.5 and 1 MCID units indicate that a treatment might be beneficial to an appreciable number of patients. Treatment effects above 1 


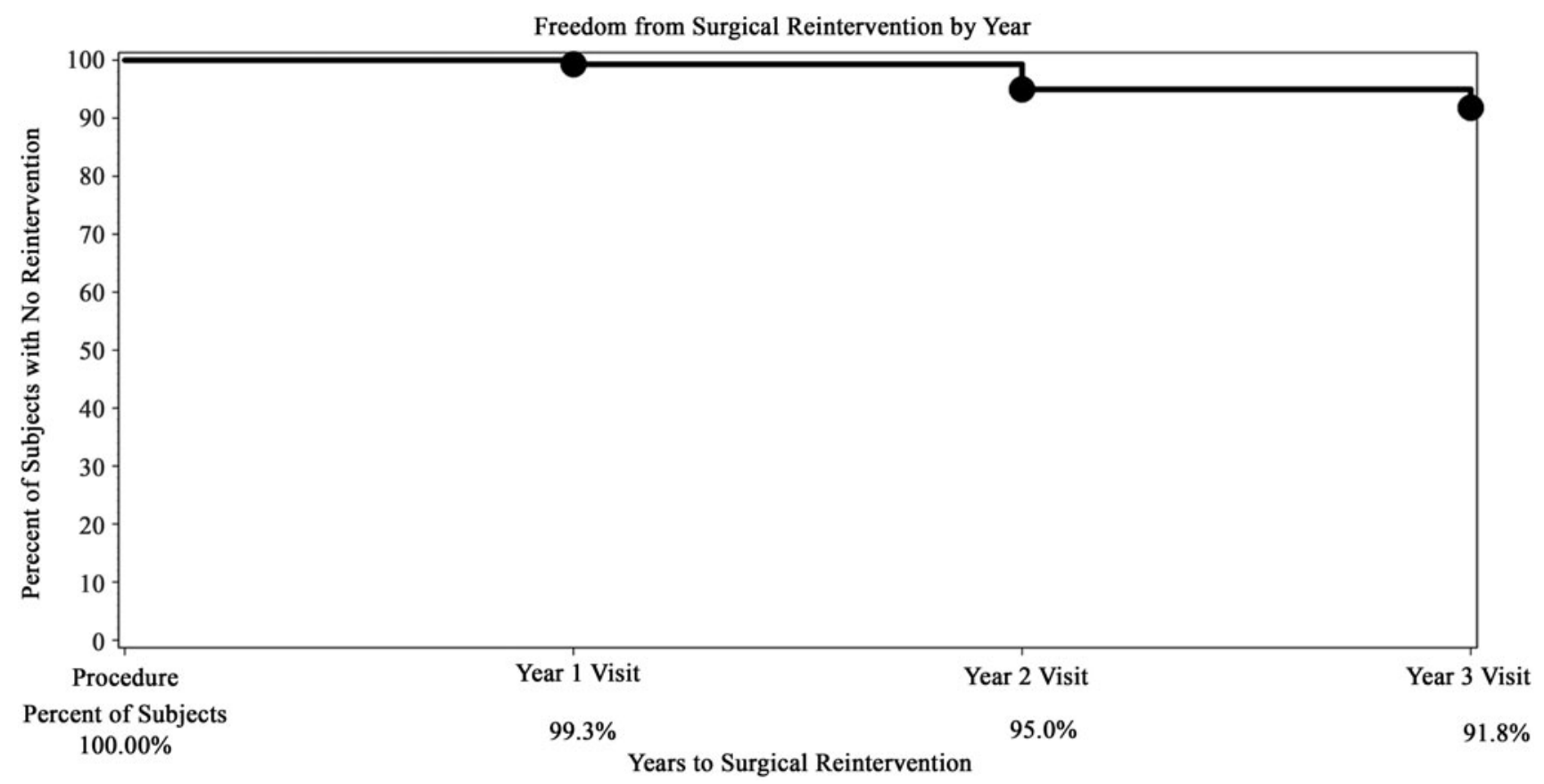

FIG. 1. Kaplan-Meier estimate of freedom from surgical reintervention due to heavy menstrual bleeding over 3 years following sonography-guided transcervical radiofrequency ablation.

MCID unit indicate that many patients might gain important benefits from treatment. ${ }^{15,16}$ Data were analyzed using SAS, version 9.3 (SAS Institute, Cary, NC, USA). All statistical tests comparing 3-year results to baseline were 2 -sided, and $p$-values $<0.05$ indicated statistically significant changes.

\section{Results}

Between April 2015 and October 2016, 147 women (mean age: 43) from 22 sites (21 in the United States, 1 in Mexico) were treated with TFA (mean: $3.0 \pm 2.1$ treated fibroids per patient; mean: $2.5 \pm 1.2 \mathrm{~cm}$ per treated fibroid). Fifteen patients were lost to follow-up during the trial, with 132 (90\%) patients accounted for at 3 years. Surgical reinterventions for HMB during follow-up included 10 hysterectomies and 1 endometrial ablation. The rates of surgical reintervention for HMB calculated with the binomial method were $0.7 \%$ at 1 year, $5.5 \%$ at 2 years, and $9.2 \%$ at 3 years during the postmarket phase. The corresponding rates using the Kaplan-Meier estimates were $0.7 \%, 5.0 \%$, and $8.2 \%$, respectively (Fig. 1).

The mean ( \pm standard deviation) SSS decreased significantly from baseline to 3 months ( $55 \pm 19$ to $27 \pm 19 ; p<0.001$ ), and this improvement was maintained durably where the final mean SSS was $22 \pm 21(p<0.001)$ at the 3-year follow-up (Fig. 2).

Similarly, HRQoL scores increased from $40 \pm 21$ at baseline to $78 \pm 22$ at 3 months $(p<0.001)$, and to $83 \pm 23$ at 3 years $(p<0.001)$ (Fig. 3). EQ-5D increased from $0.72 \pm 0.21$ at baseline to $0.87 \pm 0.13$ at 3 months $(p<0.001)$, and to $0.88 \pm 0.16$ at 3 years $(p<0.001)$ (Fig. 4$)$. When considering these treatment effects associated with TFA at each follow-up visit over 3 years in relation to established MCIDs, the improvements ranged from 2.8 to 3.2 MCID units for SSS, 1.9 to 2.2 MCID units for HRQoL, and 2.0 to 2.3 MCID units for EQ-5D (Fig. 5), indicating that, for each of these outcomes at each follow-up interval, "many patients may gain important benefits from treatment.", 15,16
At 3 years, 94\% (99/105) of patients reported satisfaction with TFA treatment ( $71 \%$ very satisfied, $14 \%$ moderately satisfied, 9\% somewhat satisfied) and $88 \%$ (92/105) of patients reported reduced fibroid symptoms, compared to baseline on the OTE questionnaire. All work productivity and activity level parameters improved significantly during the 3-year follow-up. Work absenteeism due to symptoms of uterine fibroids decreased by more than $50 \%$ (from $2.9 \%$ to $1.4 \%$ of total work time) during follow-up $(p<0.001)$. The magnitude of impairment due to uterine fibroids was dramatically reduced during the trial, with reductions from $51 \%$ to $12 \%$ for work impairment $(p<0.001)$ and from $58 \%$ to $14 \%$ for physical activity impairment $(p<0.001)$.

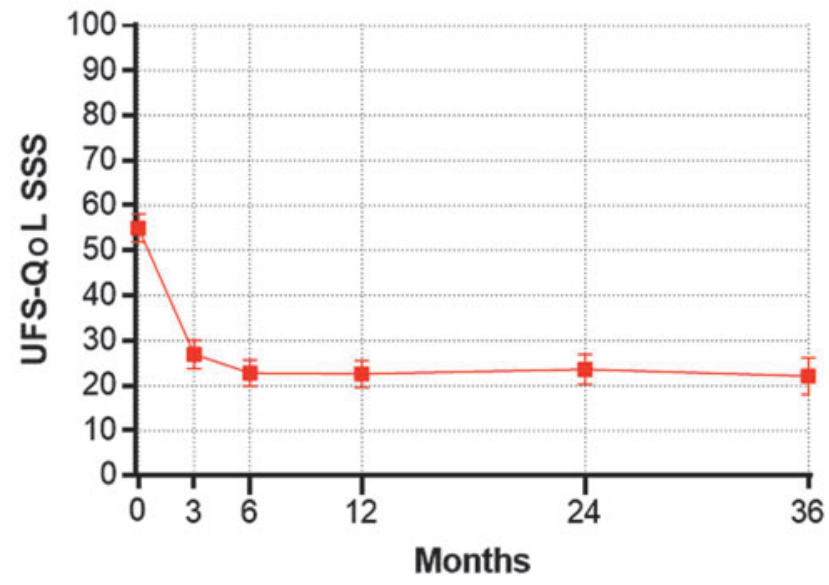

FIG. 2. Change in symptom severity score (SSS) subscale of the Uterine Fibroid Symptom and Quality-of-Life (UFSQoL) questionnaire over 3 years following sonographyguided transcervical radiofrequency ablation. Plotted values are mean and $95 \%$ confidence interval. 


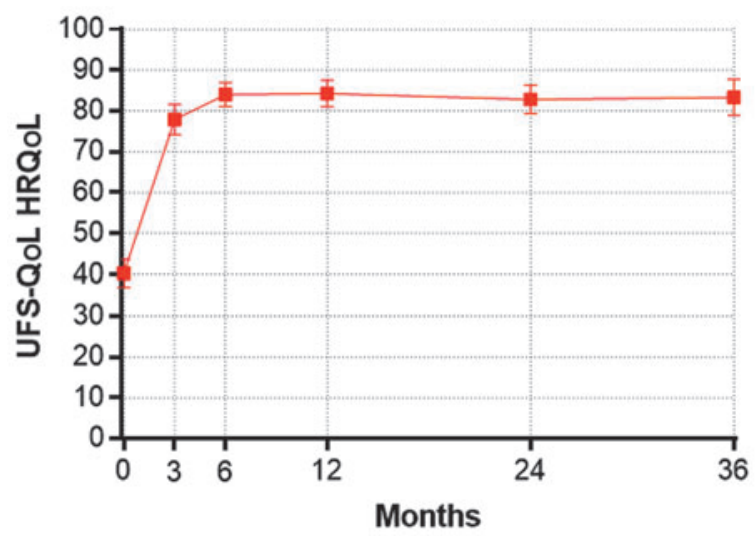

FIG. 3. Change in Health-Related Quality of Life (HRQoL) subscale of the Uterine Fibroid Symptom and Quality-of-Life (UFS-QoL) questionnaire over 3 years following sonography-guided transcervical radiofrequency ablation. Plotted values are mean and $95 \%$ confidence interval.

The 1-year safety outcomes with sonography-guided TFA in this trial were previously published, ${ }^{10}$ wherein procedurerelated serious AEs were reported in $2(1.4 \%)$ patients ( 1 with a deep venous lower-extremity thrombus and 1 with a 1-day admission for sterile leukorrhea, pelvic pain, and an unconfirmed low-grade fever), nonserious procedure-related AEs were reported in $74(50.3 \%)$ patients (most commonly leiomyoma sloughing), and no device-related AEs occurred during this time period. No serious complications or AEs related to the device or procedure occurred during the second or third year of patient follow-up. Two pregnancies were reported through 3 years. One pregnancy occurred in a 36-year-old patient who delivered at $382 / 7$ weeks gestation by elective repeat Cesarean section, 31 months after TFA. ${ }^{11}$ One miscarriage was reported in a 40-year-old subject 29 months after the Sonata treatment.

\section{Discussion}

Women who experience disruptive symptoms due to uterine fibroids comprise a challenging and underserved

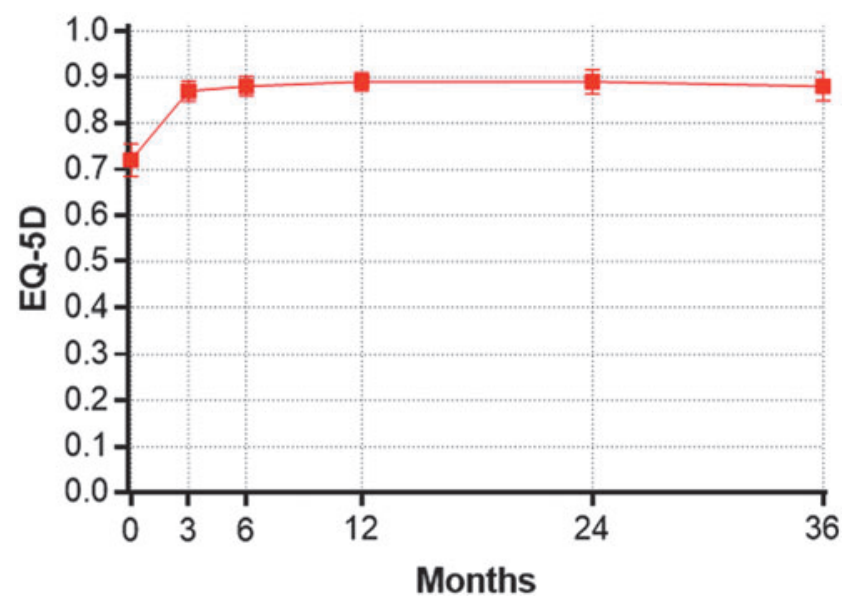

FIG. 4. Change in EuroQol 5-Dimension (EQ-5D) questionnaire score over 3 years following sonography-guided transcervical radiofrequency ablation. Plotted values are mean and $95 \%$ confidence interval. patient population. Only approximately one-third of women with symptomatic uterine fibroids seek treatment, and those who seek help often do so only after a considerable delay of nearly 4 years. ${ }^{5}$ The availability of less-invasive, uterussparing procedures might help to facilitate earlier treatment for symptomatic uterine fibroids. This article presented the 3 -year results from the SONATA trial in which 147 women with a wide variety of fibroid types were treated with TFA. Treatment with the Sonata System was shown to be safe with a low rate of procedure-related AEs, all occurring during the first year of follow-up. Furthermore, the magnitude of reduction of fibroid-related symptom severity and improvement in HRQoL was both clinically important and statistically significant at all follow-up intervals between 3 months and 3 years post-treatment. Finally, the rate of surgical reintervention for $\mathrm{HMB}$ was low and favorable, indicating that the treatment benefit was maintained durably over the 3-year follow-up time period.

There are several key attributes of TFA with the Sonata System that make it a preferable treatment option for appropriate women with symptomatic uterine fibroids. The procedure is incisionless, preserves the uterus, and has been shown to be safe and effective, producing durable symptom relief. Unlike operative hysteroscopy, TFA is designed for ablation of most nonpedunculated fibroid types. Among women treated with sonography-guided TFA in this trial, $8.2 \%$ underwent elective surgical reintervention for $\mathrm{HMB}$ over 3 years of follow-up.

These results appear to be favorable, compared to other common interventional and surgical procedures for uterine fibroids. For example, surgical reinterventions were performed in $11.0 \%$ of patients who were followed for 3 years after laparoscopic RF ablation of uterine fibroids. ${ }^{19}$ In addition, in a meta-analysis involving more than 35,000 women, surgical reintervention rates over 3 years were $9 \%$ with abdominal myomectomy, $11 \%$ with laparoscopic myomectomy, $17 \%$ with UAE, $21 \%$ with hysteroscopic myomectomy, and $24 \%$ with endometrial ablation. ${ }^{20}$

The SONATA trial design specified 3 years of patient follow-up. There is published evidence showing that surgical reintervention rates have remained low beyond 3 years following TFA. In the VITALITY study that evaluated longterm clinical outcomes of TFA over 5.4 years of mean follow-up, the surgical reintervention rate was $11.8 \% .^{21}$ Additional long-term data with the Sonata System will be available from the SAGE registry (ClinicalTrials.gov Identifier: NCT03118037), a worldwide observational postmarket study trial with the objective of characterizing long-term (5 years) outcomes after treatment of uterine fibroids with the Sonata System in real-world clinical practice settings.

The conclusions derived from this trial are robust and generalizable for several reasons. The trial enrollment included considerable ethnic and racial diversity of patients, with regional geographic representations from throughout the United States in participating centers (21 centers in the United States and 1 in Mexico). These results were achieved with 24 treating investigators, and included results from academic and community hospitals, ambulatory surgery centers, and office procedural rooms. Considering the demonstrated safety and efficacy of the Sonata treatment in a diverse patient population treated by a large number of treating physicians and different care settings, TFA is an 


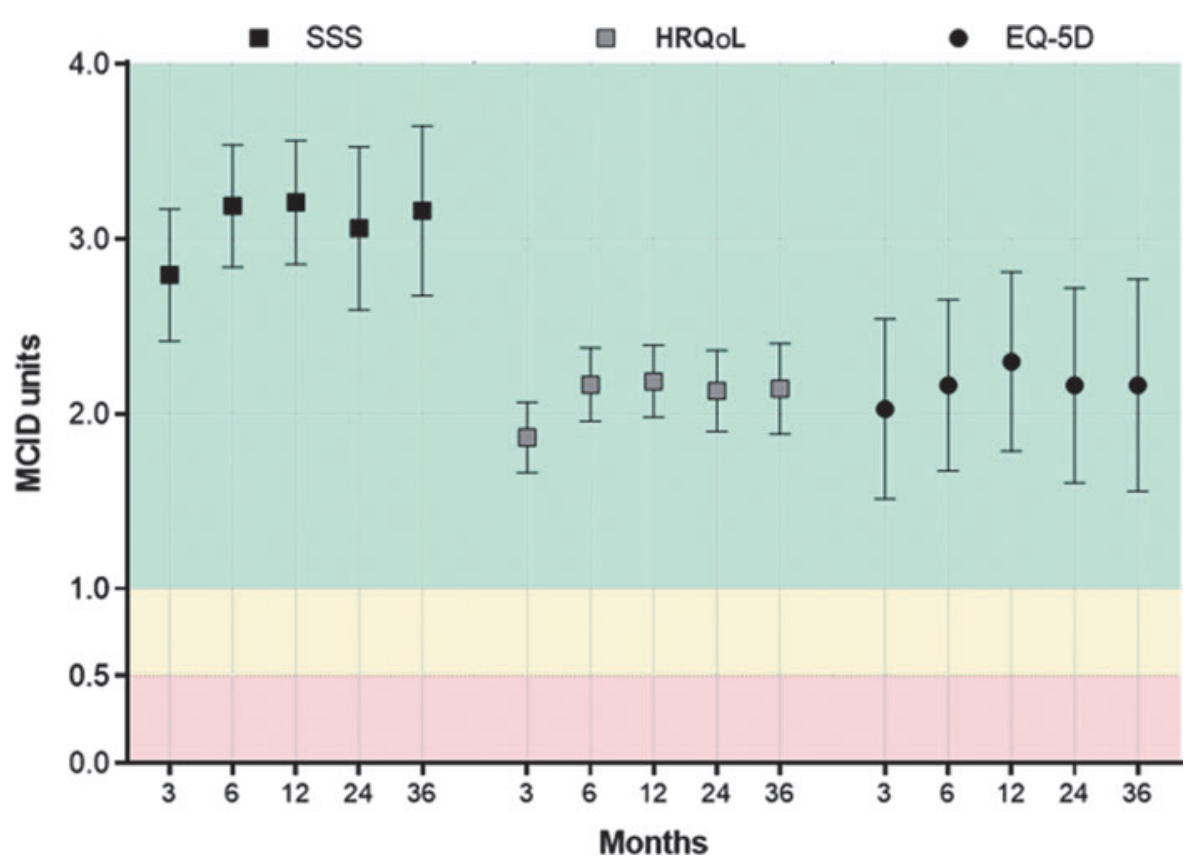

FIG. 5. Improvement in quality of life from baseline following sonography-guided transcervical radiofrequency ablation, reported in standardized minimal clinically important difference (MCID) units with 95\% confidence intervals. The MCID is 10 points for Uterine Fibroid Symptom and Quality-of-Life (UFS-QoL) Symptom Severity Score (SSS), 20 points for UFSQoL Health-Related Quality of Life (HRQoL), and 0.074 points for the EuroQol 5-Dimension (EQ-5D) questionnaire. Treatment effects below 0.5 MCID units (denoted by red background) indicate that it is unlikely that an appreciable number of patients will show a clinically important benefit. Treatment effects between 0.5 and 1 MCID units (denoted by yellow background) indicate that a treatment may be beneficial to an appreciable number of patients. Treatment effects above 1 MCID unit (denoted by green background) indicate that many patients might gain important benefits from treatment. ${ }^{15,16}$

appealing option with durable outcomes that can be included in a gynecologist's armamentarium of treatments for patients seeking treatment for symptomatic uterine fibroids.

\section{Conclusions}

Women treated with TFA for symptomatic uterine fibroids in the SONATA pivotal IDE trial experienced significant and durable reductions of fibroid-related symptoms with low surgical reintervention rates over 3 years of follow-up.

\section{Acknowledgments}

The authors acknowledge David B. Toub, MD, MBA (Gynesonics, Inc.), Taraneh G. Farazi, PhD (Gynesonics, Inc.), and Larry Miller, PhD, PStat (Miller Scientific, Johnson City, TN) for editorial assistance, and ABOND CRO Inc. (Allendale, MI) for providing data management and statistical support. The authors also thank all of the investigators and patients who participated in the SONATA clinical trial.

\section{Author Disclosure Statement}

Both authors' institutions received funding from Gynesonics, Inc. See Funding Information below.

\section{Funding Information}

Dr. Lukes: The institution of the author received research support from Gynesonics, Inc. for participation in the SONATA clinical trial. The author received travel and lodging expenses for attendance at investigator meetings.
Dr. Green: The institution of the author received research support from Gynesonics, Inc. for participation in the SONATA clinical trial. The author received travel and lodging expenses for attendance at investigator meetings.

\section{References}

1. Baird DD, Dunson DB, Hill MC, Cousins D, Schectman JM. High cumulative incidence of uterine leiomyoma in black and white women: Ultrasound evidence. Am J Obstet Gynecol 2003;188:100.

2. Zimmermann A, Bernuit D, Gerlinger C, Schaefers M, Geppert K. Prevalence, symptoms and management of uterine fibroids: An international internet-based survey of 21,746 women. BMC Womens Health 2012;12:6.

3. Foth D, Rohl FW, Friedrich C, et al. Symptoms of uterine myomas: Data of an epidemiological study in Germany. Arch Gynecol Obstet 2017;295:415.

4. Cardozo ER, Clark AD, Banks NK, Henne MB, Stegmann BJ, Segars JH. The estimated annual cost of uterine leiomyomata in the United States. Am J Obstet Gynecol 2012;206:211.e1.

5. Borah BJ, Nicholson WK, Bradley L, Stewart EA. The impact of uterine leiomyomas: A national survey of affected women. Am J Obstet Gynecol 2013;209:319 e1.

6. Bradley LD, Pasic RP, Miller LE. Clinical performance of radiofrequency ablation for treatment of uterine fibroids: Systematic review and meta-analysis of prospective studies. J Laparoendosc Adv Surg Tech A 2019;29:1507.

7. Iversen H, Dueholm M. Radiofrequency thermal ablation for uterine myomas: Long-term clinical outcomes and reinterventions. J Minim Invasive Gynecol 2017;24:1020. 
8. Lin L, Ma H, Wang J, Guan H, Yang M, Tong X, Zou Y. Quality of life, adverse events, and reintervention outcomes after laparoscopic radiofrequency ablation for symptomatic uterine fibroids: A meta-analysis. J Minim Invasive Gynecol 2019;26:409.

9. Toub DB. A new paradigm for uterine fibroid treatment: Transcervical, intrauterine sonography-guided radiofrequency ablation of uterine fibroids with the Sonata System. Curr Obstet Gynecol Rep 2017;6:67.

10. Chudnoff S, Guido R, Roy K, Levine D, Mihalov L, Garza Leal JG. Ultrasound-guided transcervical ablation of uterine leiomyomata. Obstet Gynecol 2019;133:13.

11. Miller CE, Osman KM. Transcervical radiofrequency ablation of symptomatic uterine fibroids: 2-year results of the SONATA pivotal trial. J Gynecol Surg 2019;35:345.

12. Brolmann H, Bongers M, Garza-Leal JG, Gupta J, Veersema S, Quartero R, Toub D. The FAST-EU trial: 12month clinical outcomes of women after intrauterine sonography-guided transcervical radiofrequency ablation of uterine fibroids. Gynecol Surg 2016;13:27.

13. Harding G, Coyne KS, Thompson CL, Spies JB. The responsiveness of the uterine fibroid symptom and HealthRelated Quality of Life questionnaire (UFS-HRQoL). Health Qual Life Outcomes 2008;6:99.

14. Reilly MC, Zbrozek AS, Dukes EM. The validity and reproducibility of a work productivity and activity impairment instrument. Pharmacoeconomics 1993;4:353.

15. Johnston BC, Patrick DL, Thorlund K, Busse JW, da Costa BR, Schunemann HJ, Guyatt GH. Patient-reported outcomes in meta-analyses-part 2: Methods for improving interpretability for decision-makers. Health Qual Life Outcomes 2013;11:211.
16. Johnston BC, Thorlund K, Schunemann HJ, Xie F, Murad $\mathrm{MH}$, Montori VM, Guyatt GH. Improving the interpretation of quality of life evidence in meta-analyses: The application of minimal important difference units. Health Qual Life Outcomes 2010;8:116.

17. Stewart EA, Rabinovici J, Tempany CM, et al. Clinical outcomes of focused ultrasound surgery for the treatment of uterine fibroids. Fertil Steril 2006;85:22.

18. Walters SJ, Brazier JE. Comparison of the minimally important difference for two health state utility measures: EQ5D and SF-6D. Qual Life Res 2005;14:1523.

19. Berman JM, Guido RS, Garza Leal JG, Pemueller RR, Whaley FS, Chudnoff SG, Halt Study G. Three-year outcome of the HALT trial: A prospective analysis of radiofrequency volumetric thermal ablation of myomas. J Minim Invasive Gynecol 2014;21:767.

20. Davis MR, Soliman AM, Castelli-Haley J, Snabes MC, Surrey ES. Reintervention rates after myomectomy, endometrial ablation, and uterine artery embolization for patients with uterine fibroids. J Womens Health (Larchmt) 2018;27:1204.

21. Garza-Leal JG. Long-term clinical outcomes of transcervical radiofrequency ablation of uterine fibroids: The VITALITY study. J Gynecol Surg 2019;35:19.

Address correspondence to: Andrea Lukes, MD Carolina Women's Research and Wellness Center 249 East NC Highway 54, Suite 330 Durham, NC 27713

USA

E-mail: andrealukes@cwrwc.com 